\title{
Rapid one-step protein purification from plant material using the eight-amino acid StrepII epitope ${ }^{\star}$
}

\author{
Claus-Peter Witte*, Laurent D. Noël ${ }^{1}$, Janine Gielbert, Jane E. Parker and Tina Romeis \\ Department of Plant-Microbe Interactions, Max-Planck-Institute for Plant Breeding Research, Carl-von- \\ Linné-Weg 10, 50829 Cologne, Germany (*author for correspondence; e-mail witte@mpiz-koeln.mpg.de) \\ ${ }^{1}$ Present address: Laboratoire de Biologie du Développement des plantes, UMR CNRS-CEA-Université \\ méditerranée 6191, DEVM, CEN Cadarache, 13108 Saint Paul lez Durance Cedex, France
}

Received 26 March 2004; accepted in revised form 2 June 2004

Key words: affinity purification, chitin-binding domain tag, HAT tag, S tag, StrepII tag, TAP tag

\begin{abstract}
Beyond the rewards of plant genome analysis and gene identification, characterisation of protein activities, post-translational modifications and protein complex composition remains a challenge for plant biologists. Ideally, methods should allow rapid isolation of proteins from plant material achieving a high degree of purity. We tested three purification strategies based on the eight-amino acid StrepII, six-amino acid $\mathrm{His}_{6}$ and 181-amino acid Tandem Affinity Purification (TAP) affinity tags for enrichment of a membraneanchored protein kinase, $N t \mathrm{CDPK} 2$, and a soluble protein, $A t \mathrm{SGT} 1 \mathrm{~b}$, from leaf extracts. Transiently expressed StrepII-tagged $N t$ CDPK2 was purified from Nicotiana benthamiana to almost complete homogeneity in less than $60 \mathrm{~min}$ and was directly suitable for enzymatic or mass-spectrometric analyses, allowing the identification of in planta phosphorylation sites. In contrast, purification of $\mathrm{NtCDPK}_{2} \mathrm{via} \mathrm{His}_{6} \mathrm{tag}$ yielded partially oxidised protein of low purity. AtSGT1b could be isolated after transient expression from $N$. benthamiana or from transgenic Arabidopsis thaliana as either TAP-tagged or StrepII-tagged protein. While StrepII-tag purification achieved similar yield and high purity as the TAP-tag strategy, it was considerably easier and faster. Using either tagging strategy, a protein was co-purified with $A t \mathrm{SGT} 1 \mathrm{~b}$ from $N$. benthaniana and $A$. thaliana leaf extracts, suggesting that both the StrepII and TAP tags are suitable for purification of protein complexes from plant material. We propose that the StrepII epitope, in particular, may serve as a generally utilizable tag to further our understanding of protein functions, post-translational modifications and interaction dynamics in plants.
\end{abstract}

Abbreviations: AEBSF, 4-(2-aminoethyl)benzenesulfonyl fluoride hydrochloride; AP, alkaline phosphatase; CDPK, calcium dependent protein kinase; HA, haemaglutinin; IMAC, immobilised metal affinity chromatography; Q-TOF MS, quadrupole time-of-flight mass spectrometry; TAP tag, tandem affinity purification tag

\section{Introduction}

An increasing interest in analysing the biochemical functions, post-translational modifications and

\footnotetext{
* The first two authors contributed equally to this work.
}

interacting partners of plant proteins demands improved purification techniques. Efraim Racker's motto "Don't waste clean thinking on dirty enzymes" adopted by Arthur Kornberg in his "ten commandments" (Kornberg, 2003) may serve as a guide. But how do we best get "dirty" proteins clean? This is greatly facilitated by the 
use of affinity tags. These polypeptides or small proteins are fused to the protein of interest and allow purification via an affinity matrix. A wide variety of affinity tags is currently available (Terpe, 2003). The ideal tag is small and allows rapid and flexible purification from a complex mixture, achieving high yield and purity. A small tag is generally less likely to interfere with the biological function of a protein. Rapid purification aids in maintaining protein integrity and post-translational modifications and increases the probability of co-purifying transiently bound interactors. Flexibility ensures that starting materials and buffers as well as buffer additives are not limited by the particular requirements of the tag. High yield and purity facilitate biochemical characterisation and make it easier to distinguish interactors from background.

In purifiying two different proteins from leaf crude extracts, we tested the performance of the StrepII (WSHPQFEK), the $\mathrm{His}_{6}$ and the tandem affinity purification-(TAP) tags. The StrepII tag binds to a streptavidin derivative termed Strep Tactin. It mimics structurally biotin and can be eluted from the StrepTactin matrix by washing with biotin or desthiobiotin containing buffers (Skerra and Schmidt, 2000; www.iba-go.com). The $\mathrm{His}_{6}$ tag, comprising a stretch of six consecutive histidines, allows purification via immobilised metal affinity chromatography (IMAC). Nickel or Cobalt chelating resins are used (Chaga, 2001). The TAP tag has an IgG-binding protein A moiety separated by a TEV protease cleavage site from a calmodulin-binding peptide. The tagged protein is first bound via its protein $\mathrm{A}$ to an IgG column, cleaved off using TEV protease and then bound to a calmodulin column in the presence of calcium and eluted with EGTA (Rigaut et al., 1999).

In this study we were aiming to find methods for rapid isolation of proteins in high purity for subsequent mass-spectrometric analyses. We purified the membrane-associated calcium-dependent protein kinase $N t$ CDPK2 from tobacco (Romeis et al., 2001), transiently expressed in $N$. benthamiana, and the soluble protein $A t \mathrm{SGT} 1 \mathrm{~b}$ from A. thaliana (Austin et al., 2002) either transiently expressed in $N$. benthamiana or stably expressed in transgenic A. thaliana. NtCDPK2StrepII purification was compared to $N t$ CDPK2$\mathrm{His}_{6}$ purification. For AtSGT1b we compared purifications via the StrepII tag and the TAP tag. These experiments demonstrate that the StrepII tag is vastly superior to $\mathrm{His}_{6}$ in terms of flexibility and purity and offers key advantages over the TAP tag in terms of size, speed of purification and flexibility. We conclude that the StrepII tag is a valuable tool for rapid, easy and high quality protein purification from plant material.

\section{Materials and methods}

\section{Vectors, bacterial strains, plant material and} growth conditions

The following binary vectors derived from pamPAT-MCS (accession number AY436765) were used in this study: pXCS-GFP, pXCS-HisHA, pXCS-HAHAT, pXCS-HAStrep (accession number AY457636), pXCSG-Strep and pXCSGTAP (see below for construction details). These were introduced into Agrobacterium tumefaciens GV3101::pMP90RK (Koncz and Schell, 1986) carrying gentamycin, kanamycin and rifampicin resistances and conferred an additional carbenicillin resistance to transformed bacteria. The binary vector 35S:p19 conferring kanamycin resistance and carrying the gene for the p19 silencing suppressor from tomato bushy stunt virus was present in A. tumefaciens $\mathrm{C} 58 \mathrm{Cl}$ (possessing rifampicin resistance) which carried the helper plasmid $\mathrm{pCH} 32$ (tetracyclin resistance) for T-DNA transfer (Voinnet et al., 2003). Agrobacterium strains were grown at $28^{\circ} \mathrm{C}$ in YEB medium. Antibiotics were added to the media at the following final concentrations: carbenicillin, $75 \mu \mathrm{g} / \mathrm{ml}$ (solid), $50 \mu \mathrm{g} / \mathrm{ml}$ (liquid); gentamycin, $15 \mu \mathrm{g} / \mathrm{ml}$; kanamycin, $50 \mu \mathrm{g} / \mathrm{ml} ; \quad$ rifampicin, $100 \mu \mathrm{g} / \mathrm{ml}$, tetracyclin $10 \mu \mathrm{g} / \mathrm{ml}$. Agrobacteria were grown first on solid media and densely inoculated from fresh plates into liquid media to achieve sufficient growth within 16 to $20 \mathrm{~h}$. Longer growing times led to reduced expression in planta.

Arabidopsis Landsberg erecta sgt1b-3 null mutant plants (Austin et al., 2002) were grown at $21^{\circ} \mathrm{C}, \quad 10 \mathrm{~h}$ light period at $150-200 \mu$ Einsteins $\cdot \mathrm{m}^{-2} \cdot \mathrm{sec}^{-1}$ (intensity) and $60 \%$ humidity. $N$. benthamiana were grown in late summer in a climatised greenhouse at $21{ }^{\circ} \mathrm{C}$ and $16 \mathrm{~h}$ light. 
Generation of the $p X C$-tag derivatives

To generate pXCS-HAStrep (accession number AY457636), the adapter composed of $\underline{\mathrm{CCC}}$ GGGGTTATCCATACGATGTTCCAGATTAT GCTGTCGGCGCCGGTTGGTCTCATCCTCA ATT TGAAAAATAAGTCTAGA (XmaI and $X b a I$ sites underlined) was ligated into pamPATMCS (accession number AY436765) digested by $X m a I$ and $X b a I$. Similarly, to obtain pXCS-HisHA, the adapter composed of CCCGGGATAATGGC TCATCACCATCACCATCATGGCGCCTATC CATACGATGTTCCAGATTATGCTG TCT AGA was inserted into pamPAT-MCS. NtCDPK2 was amplified from cloned cDNA (Romeis et al., 2001) with primers GAATTCAAAATGGGGAAC ACTTG and CCCGGGAAGTCTTAGAGCCTC and cloned directionally into the EcoRI and $X m a \mathrm{I}$ sites of either pXCS-HAStrep or pXCS-HisHA, resulting in $\mathrm{pXCS}-\mathrm{NtCDPK} 2-\mathrm{HAStrep}$ and $\mathrm{pXCS}$ NtCDPK2-HisHA. Cloning details for pXCSNtCDPK2-HAHAT ( $N$ CDPK2 with HA and HAT tags) are available upon request.

To generate pXCS-GFP, the $s m G F P$ ORF was amplified from psmGFP (accession number U70495; Davis and Vierstra, 1996) using the primers ATCCCCGGGATGAGTAAAGGAGA AGAACTTTTC and AGTCTAGAGCTCTTAT TTGTATAGTTCATCCATG and cloned directionally into the $X m a I$ and $X b a I$ sites of pXCSHAStrep. To construct pXCS-TAP, the TAP ORF was amplified from pBS1479 (Puig et al., 2001) using the primers GCCCCGGGTTAG CAGAAGCTAAAAAGCTA and TCTCTAGA ATCAAGCTTCAGGTTGACTTC and cloned directionally into the $X m a \mathrm{I}$ and $X b a \mathrm{I}$ sites of pXCS-HAStrep. pXCSG-TAP and pXCSG-Strep were generated by ligation of the GATEWAY reading frame $\mathrm{B}$ (rfB; Blunt $E c o \mathrm{RV}$ fragment, Invitrogen, Carlsbad, CA) into pXCS-TAP digested with SmaI and pXCS-HAStrep, digested by $S f o \mathrm{I} / S m a \mathrm{I}$, respectively. AtSGTIb cDNA from the Ler ecotype was amplified from pCA134 (Azevedo et al., 2002) using CACCATGGCCAAGGAATTAGCAG and ATACTCCCACTTCTTGAGCTCCATG, cloned directionally into pENTR/D-TOPO (Invitrogen) and recombined by $\mathrm{LR}$ reaction following the manufacturer's instructions into pXCSG-TAP and $\mathrm{pXCSG}$-Strep giving $\mathrm{pXCSG-SGT1b-TAP}$ and pXCSG-SGT1b-Strep, respectively.
Transient protein expression in $\mathrm{N}$. benthamiana

Experiments were performed as described (Romeis et al., 2001). Agrobacterium cells in late exponential phase $(<20 \mathrm{~h}$ growth in liquid medium) were harvested by centrifugation and resuspended in $10 \mathrm{mM}$ MES-KOH (pH 5.6) containing $10 \mathrm{mM}$ $\mathrm{MgCl}_{2}$ and $150 \mu \mathrm{M}$ acetosyringone to an $\mathrm{OD}_{600 \mathrm{~nm}}$ of $\sim 0.9$. These cells were mixed with a dense solution of $\mathrm{C} 58 \mathrm{Cl}$ (pCH32 35S:p19) expressing the silencing suppressor p19 of tomato bushy stunt virus (Voinnet et al., 2003) so that the final density of the $\mathrm{C} 58 \mathrm{C} 1$ cells was about 0.6. Bacteria were incubated for $2 \mathrm{~h}$ at room temperature and then injected into young fully expanded leaves of 4-weeks-old $N$. benthamiana plants. Leaves were harvested after 4 days, frozen in liquid nitrogen and stored at $-80{ }^{\circ} \mathrm{C}$.

\section{Stable transformation of Arabidopsis}

The Landsberg erecta sgt $1 b-3$ null mutant plants (Austin et al., 2002) were transformed by flower dipping as described (Clough and Bent, 1998). Transformants were selected by spraying sevenday-old seedlings with $0.1 \%$ Basta (Aventis). Basta-resistant lines expressing detectable amounts of the AtSGT1b-tag variants were self pollinated to produce T2 seeds. Single insertion lines were selected by segregation analysis of the Basta resistance in the T2 population. Leaves from twoto three-weeks-old Basta-resistant T2 plants were harvested, frozen in liquid nitrogen and stored at $-80{ }^{\circ} \mathrm{C}$.

\section{Purification via the StrepII tag}

Leaf material $(0.25 \mathrm{~g}$ and $0.75 \mathrm{~g}$ for $N$. benthamiana and $A$. thaliana, respectively) was ground in liquid nitrogen and thawed in $0.75 \mathrm{ml} \mathrm{Ex}$-strep buffer (100 mM Tris, pH 8.0; $5 \mathrm{mM}$ EGTA; $5 \mathrm{mM}$ EDTA; $150 \mathrm{mM} \mathrm{NaCl} ; 10 \mathrm{mM}$ DTT; $0.5 \mathrm{mM}$ AEBSF (4-(2-aminoethyl)benzenesulfonyl fluoride hydrochloride); $5 \mu \mathrm{g} / \mathrm{ml}$ aprotinin; $5 \mu \mathrm{g} / \mathrm{ml}$ leupeptin; plant protease inhibitor cocktail (Sigma P9599, Taufkirchen, Germany), diluted 1:200; $0.5 \%$ Triton X-100; and $100 \mu \mathrm{g} / \mathrm{ml}$ avidin). The slurry (about $0.8 \mathrm{ml}$ ) was placed in a $2 \mathrm{ml}$ microfuge tube, transferred for $30 \mathrm{~s}$ into a sonication bath and then centrifuged for $10 \mathrm{~min}$ at $4{ }^{\circ} \mathrm{C} \quad(21000 \mathrm{~g})$. The supernatant was 
transferred to a new microfuge tube, sampled (lanes 1 of Figures 2A and 6A), and $150 \mu$ slurry of StrepTactin Sepharose or StrepTactin Macroprep (IBA GmbH, Göttingen, Germany) was added. The two resins differ in the matrix to which the functional StrepTactin groups are attached: the Sepharose matix is based on Sepharose $4 \mathrm{FF}$ with a bead size of $45-165 \mu \mathrm{m}$ while the Macroprep matrix consists of polymethacrylate beads with a diameter of $50 \mu \mathrm{m}$. All samples taken for electrophoretic analysis were mixed with a $3 \times$ SDS-loading buffer and heated for $3 \mathrm{~min}$ to $90{ }^{\circ} \mathrm{C}$ prior to loading. Binding was performed by incubation in an end-over-end rotation wheel for 30 min at $4{ }^{\circ} \mathrm{C}$. The slurry was transferred into a micro spin column (BioRad 732-6204, Hercules, $\mathrm{CA})$ and the flow-through collected and sampled (lanes 2). The resin was washed twice with $1 \mathrm{ml}$ and four times with $0.5 \mathrm{ml} \mathrm{W}$-strep buffer (50 mM Tris, $\mathrm{pH}$ 8.0; $2.5 \mathrm{mM}$ EDTA; $150 \mathrm{mM}$ $\mathrm{NaCl}$; 2 mM DTT; $0.05 \%$ Triton X-100). To allow withdrawal of a sample before elution (lanes 3 ), the resin was resuspended in $0.8 \mathrm{ml} \mathrm{W}$-strep buffer (same volume as original extract to facilitate comparisons). After taking a sample of the slurry (buffer including beads), the W-strep buffer was drained off again. For elution, $80 \mu \mathrm{l}$ of Ebuffer $(10 \mathrm{mM}$ Tris $\mathrm{pH} 8.0 ; 10 \mathrm{mM}$ desthiobiotin; $2 \mathrm{mM}$ DTT; $0.05 \%$ Triton X-100) representing the void volume of the system were carefully applied to the resin but not recovered. Four times $100 \mu \mathrm{l}$ E-buffer were passed through and collected in two pools of $200 \mu \mathrm{l}$ each (lanes 4 and 5). The resin was washed with $0.5 \mathrm{ml} \mathrm{W}$-strep buffer and resuspended in $0.8 \mathrm{ml} \mathrm{W}$-strep buffer for taking a slurry sample after elution (lanes 6). In case of subsequent direct Q-TOF MS analysis Triton X-100 needed to be removed completely. To achieve this, at least three washes without detergent were performed prior to elution. If Macroprep resin was used, one volume of washed beads was resuspended in one volume of $2 \times$ SDS-gel loading buffer and heated to $90{ }^{\circ} \mathrm{C}$ for $6 \mathrm{~min}$. The liquid phase (without beads) was loaded on SDS gels. For assessment of protein kinase activity, $1 \mu \mathrm{l}$ kinase of the first elution pool was incubated with $10 \mu \mathrm{M}$ syntide- 2 and $10 \mu \mathrm{M}\left[\gamma^{32} \mathrm{P}\right]$ ATP $\left(10 \mu \mathrm{Ci} \mathrm{nmol}^{-1}\right)$ in the presence of $1 \mathrm{mM} \mathrm{CaCl}_{2}$ or $2 \mathrm{mM}$ EGTA for $5 \mathrm{~min}$ at $30^{\circ} \mathrm{C}$ (Romeis et al., 2001). The phosphorylated substrate was quantified by scintillation counting.

\section{Purification via $\mathrm{His}_{6}$ or HAT tag}

The leaf material $(0.25 \mathrm{~g}$ per purification $)$ was ground in liquid nitrogen and thawed in $0.75 \mathrm{ml}$

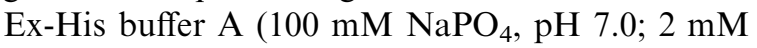
EGTA; $2 \mathrm{mM}$ EDTA; $150 \mathrm{mM} \mathrm{NaCl} ; 2 \mathrm{mM}$ DTT; $0.5 \mathrm{mM}$ AEBSF; $5 \mu \mathrm{g} / \mathrm{ml}$ aprotinin; $5 \mu \mathrm{g} /$ $\mathrm{ml}$ leupeptin; plant protease inhibitor cocktail (Sigma P9599), diluted 1:200). The slurry (about $0.8 \mathrm{ml}$ ) was placed in a $2 \mathrm{ml}$ microfuge tube and centrifuged for $10 \mathrm{~min}$ at $4{ }^{\circ} \mathrm{C}(21000 \mathrm{~g})$. The pellet was resuspended in $0.75 \mathrm{ml} \mathrm{Ex}$-His buffer B (as buffer A but lacking EGTA, EDTA, DTT and inhibitor cocktail) and centrifuged for $5 \mathrm{~min}$ at $4{ }^{\circ} \mathrm{C}$. The pellet was resuspended in $0.75 \mathrm{ml}$ Ex-His buffer B containing $0.5 \%$ Triton X-100, transferred for $30 \mathrm{~s}$ into a sonication bath and centrifuged for $5 \mathrm{~min}$ at $4{ }^{\circ} \mathrm{C}$. The supernatant was recovered, sampled (lane 1 of Figure 2B), and $150 \mu \mathrm{l} 50 \%$ Talon slurry (BD Biosciences Clontech), previously washed with Ex-His buffer $\mathrm{B}$, was added. The slurry was incubated for $30 \mathrm{~min}$ at $4{ }^{\circ} \mathrm{C}$ on a rotation wheel, transferred into a micro spin column and the flow-through was collected and sampled (lane 2). All following steps were performed as described for the purification via StrepII tag, except that other buffers were used. The wash buffer consisted of $50 \mathrm{mM}$ $\mathrm{NaPO}_{4}, \mathrm{pH} 7.0 ; 300 \mathrm{mM} \mathrm{NaCl}$; and $0.05 \%$ Triton $\mathrm{X}-100.150 \mathrm{mM}$ imidazole was added to the wash buffer for elution.

\section{Purification via TAP tag}

Leaf material $(0.25 \mathrm{~g}$ and $0.75 \mathrm{~g}$ for $N$. benthamiana and $A$. thaliana, respectively) was ground in liquid nitrogen and thawed in $0.75 \mathrm{ml}$ Ex-strep buffer without avidin. The slurry (about $0.8 \mathrm{ml}$ ) was placed in a $2 \mathrm{ml}$ microfuge tube, transferred for $30 \mathrm{~s}$ into a sonication bath and then centrifuged for $10 \mathrm{~min}$ at $4{ }^{\circ} \mathrm{C}(21000 \mathrm{~g})$. Purification was essentially performed as described (Rigaut et al., 1999). The supernatant was transferred in a new centrifuge tube, sampled (lane 1 of Figure $6 \mathrm{~B}$ ), and $150 \mu \mathrm{l}$ of washed rabbit $\mathrm{IgG}$ agarose beads (Sigma A2909) was added followed by an incubation for $1 \mathrm{~h}$ at $4{ }^{\circ} \mathrm{C}$ on an end-overend rotation wheel. The slurry was transferred into a micro-spin column and the flow-through was sampled (lane 2). The resin was washed twice with $1 \mathrm{ml}$ and four times with $0.5 \mathrm{ml} \mathrm{W}$-TAP buffer 
(50 mM Tris, pH 8.0; 5 mM EDTA; $5 \mathrm{mM}$ EGTA; $150 \mathrm{mM} \mathrm{NaCl} ; 2 \mathrm{mM}$ DTT; $0.05 \%$ Triton $\mathrm{X}-100$ ). To withdraw a sample before elution (lane 3 ), the resin was resuspended in $0.8 \mathrm{ml} \mathrm{W}$-TAP buffer. (same volume as original extract to facilitate comparisons). After taking a sample of the slurry, the W-TAP buffer was drained off again. The column was first equilibrated with $1 \mathrm{ml}$ and two times $0.5 \mathrm{ml}$ TEV buffer $(50 \mathrm{mM}$ Tris, $\mathrm{pH} 8.0$; $0.5 \mathrm{mM}$ EDTA; $150 \mathrm{mM} \mathrm{NaCl} ; 2 \mathrm{mM}$ DTT; $0.05 \%$ Triton $\mathrm{X}-100$ ). TEV protease digest was performed at $16{ }^{\circ} \mathrm{C}$ for $2 \mathrm{~h}$ in $500 \mu \mathrm{l} \mathrm{TEV}$ buffer with 100U TEV protease (Invitrogen). The flowthrough was collected (lane 4) and added to $3 \mu \mathrm{l}$ $1 \mathrm{M} \mathrm{CaCl}_{2}, 1.5 \mathrm{ml}$ Cam Binding Buffer $(50 \mathrm{mM}$ Tris, $\mathrm{pH} 8.0 ; 150 \mathrm{mM} \mathrm{NaCl} ; 10 \mathrm{mM} \beta$-mercaptoethanol; $1 \mathrm{mM}$ Mg-acetate; $1 \mathrm{mM}$ imidazole; $2 \mathrm{mM} \mathrm{CaCl}_{2} ; 0.05 \%$ Triton X-100) and $150 \mu \mathrm{l}$ washed calmodulin agarose beads (Stratagene \#214303, Amsterdam-Zuidoost, The Netherlands). After $1 \mathrm{~h}$ at $4{ }^{\circ} \mathrm{C}$ on a rotation wheel, the slurry was transferred into a micro spin column (flowthrough, lane 6). To collect a sample before elution, the resin was resuspended in $0.8 \mathrm{ml}$ Cam Binding Buffer (lane 7) and the buffer was drained off after sampling. The final steps were performed as described for the StrepII-tag elution using the Cam E-buffer (50 mM Tris, pH 8.0; $150 \mathrm{mM}$ $\mathrm{NaCl} ; 10 \mathrm{mM} \beta$-mercaptoethanol; $1 \mathrm{mM} \mathrm{Mg-}$ acetate; $1 \mathrm{mM}$ imidazole; $2 \mathrm{mM}$ EGTA; $0.05 \%$ Triton X-100).

\section{SDS-gel electrophoresis, Western blot analysis and protein stainings}

Proteins were separated by SDS-PAGE on $10 \%$ acrylamide gels and transferred onto nitrocellulose membranes by tank blotting (Tris-glycine buffer, $\left.\mathrm{pH} 8.3 ; 20 \mathrm{~V} / \mathrm{cm}^{1} ; 1 \mathrm{~h}\right)$. Membranes were blocked in $5 \%$ milk powder in TBS buffer containing $0.1 \%$ Tween 20 (TBS-T buffer) for $30 \mathrm{~min}$. Prior to the incubation with the StrepTactin-alkaline phosphatase (AP) conjugate, the blots were rinsed several times in TBS-T to remove free biotin originating from the milk powder, followed by a $10 \mathrm{~min}$ pre-incubation with TBS-T containing $2 \mu \mathrm{g} / \mathrm{ml}$ avidin (Fluka 11368, Buchs, Switzerland) to block biotinylated proteins on the blot. StrepTactin-AP conjugate was then added to the pre-incubation solution. For detection with the Streptavidin-AP conjugate, the blots were also rinsed with TBS-T but not blocked with avidin. Antibodies and alkaline phosphatase conjugates were used in the following dilutions: StrepTactinAP conjugate (IBA 2-1503-001, Göttingen, Germany) 1:4 000; Streptavidin-AP conjugate (Calbiochem OR04L, San Diego, CA) 1:2000; rat anti-HA antibody (Roche 1867 423, Mannheim, Germany) 1:4000; rat anti-SGS antibody (raised against AtSGT1a SGS domain; S. Betsuyaku et al., unpublished); goat anti-rat IgG AP conjugate (Sigma A-9654) 1:10 000. First antibodies and conjugates were incubated at room temperature for $1-2 \mathrm{~h}$ or $\mathrm{o} / \mathrm{n}$ at $4{ }^{\circ} \mathrm{C}$ followed by three $10 \mathrm{~min}$ washes with TBS-T and a $1 \mathrm{~h}$ incubation with the secondary antibody where applicable. After three 10 min TBS-T washes, staining was performed with p-nitro blue tetrazolium.

Coomassie stains were performed with $0.25 \%$ Coomassie $\mathrm{R}$ in 45:45:10 water, methanol, acetic acid. Silver staining compatible with mass spectrometry was performed as described (Shevchenko et al., 1996).

\section{Mass spectrometry}

Tryptic digestion of StrepII-purified proteins was either performed directly in solution or in-gel after SDS-PAGE using a protocol based on Shevchenko et al. (2000). Digests were desalted for electrospray MS with a $\mathrm{C} 18$ reverse phase resin (ZipTip, Millipore, Bedford, MA). A Micromass Q-Tof-2 mass spectrometer operating with nanospray and the Masslynx software (version 3.5) were used for analysis.

\section{Results}

Construction of $p X C$ binary vectors for expression of $C$-terminally tagged proteins in planta

A family of binary vectors based on the pamPATMCS backbone (accession number AY436765) was generated to allow convenient and rapid in planta expression of proteins with various tags or promoters (see Materials and methods for cloning details). These vectors are high copy number in $E$. coli and confer ampicillin and carbenicillin resistance to $E$. coli and A. tumefaciens, respectively. The pat gene confers Basta herbicide resistance allowing rapid selection of transgenic 

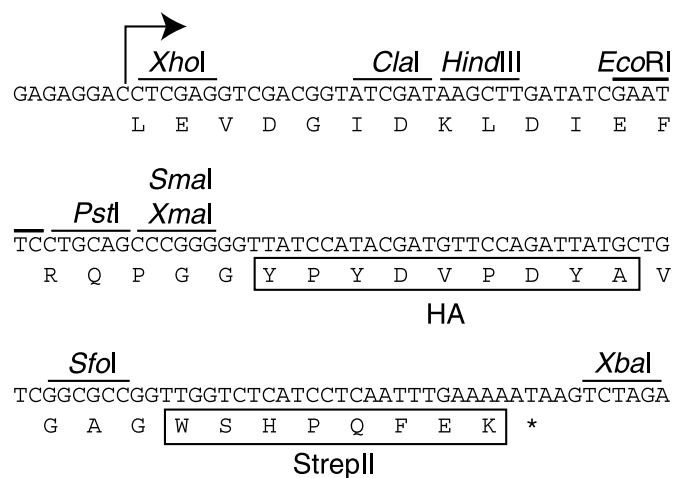

Figure 1. Polylinker map of pXCS-HAStrep. The nucleotide sequence of pXCS-HAStrep (accession number AY457636, base pairs 5292 to 94 ) is shown with the relevant unique restriction sites and coding frame. Boxed amino acids indicate the HA and StrepII epitopes. The $35 \mathrm{~S}$ transcription start is represented by an arrow.

plants on soil. A series of derivatives of this vector with different tags, promoters and other features were generated and the following nomenclature adopted: "pX" (backbone); "C or N" (position of the tag relative to the protein sequence, here only C); "S" $(2 \times 35 \mathrm{~S}$ promoter); "G" (GATEWAYcompatible); "tags". As an example, the complete sequence of pXCS-HAStrep has been deposited in the DNA database (Figure 1; accession number AY457636). In this study, we used pXCS-HAStrep and pXCS-HisHA for cloning of NtCDPK2 and the GATEWAY-compatible vectors pXCSGStrep and pXCSG-TAP for cloning of AtSGT1b. The $\mathrm{pXCS}-\mathrm{GFP}$ vector was used as control.

\section{StrepII-tag and His6-tag purifications of $\mathrm{NtCDPK2}$}

CDPKs are serine/threonine protein kinases unique to plants that bind calcium at a C-terminal calmodulin-like domain. N-terminal myristoylation and palmitoylation leads to the association of many CDPKs with cellular membranes (Cheng et al., 2002). NtCDPK2 was found to play an essential role in plant defence response (Romeis et al., 2001).

$N t$ CDPK2-HA-StrepII was isolated from crude $N$. benthamiana leaf extracts to high purity using StrepTactin Sepharose (Figure 2A). Starting from the crude extract (lane 1), the purification was performed in less than 60 min, yielding a single band in Coomassie-stained or silver-stained gels (lanes 4 and 7, respectively). The purified protein was active, possessing calcium-dependent
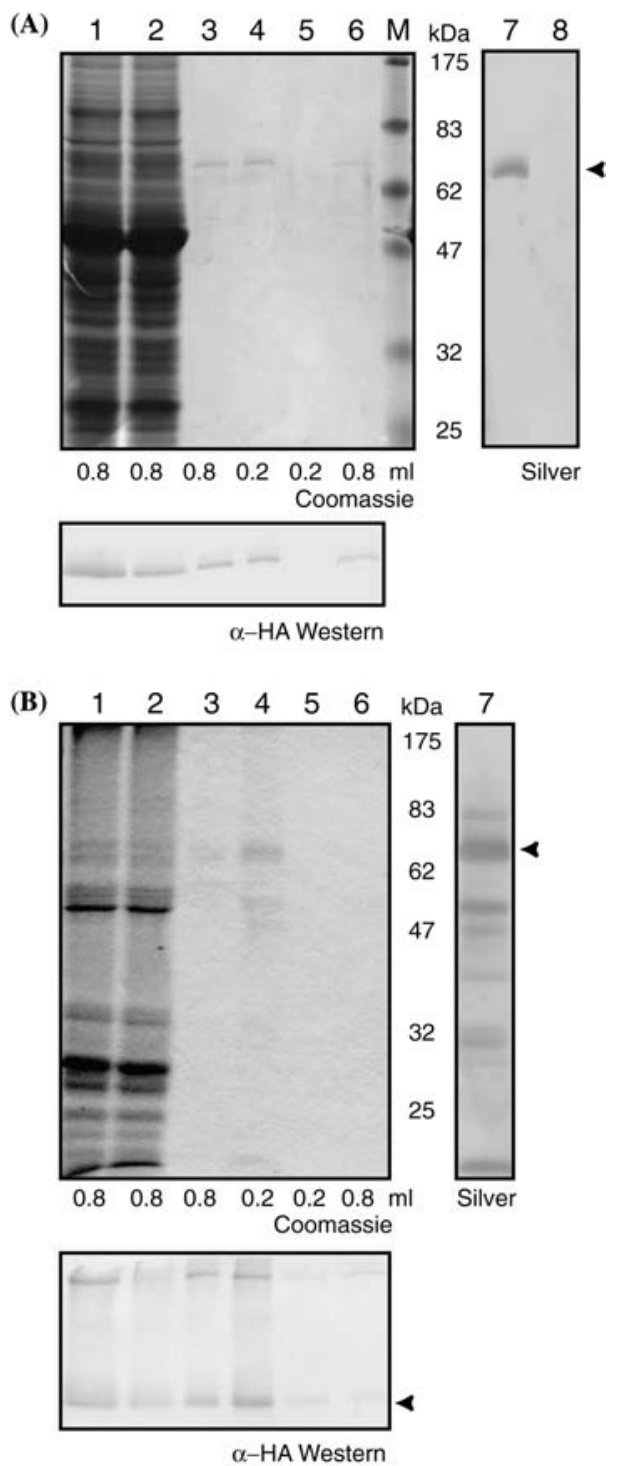

Figure 2. $N t$ CDPK2 purification from $N$. benthamiana via StrepII or $\mathrm{His}_{6}$ tag. The different fractions collected during purification of $\mathrm{NtCDPK} 2-\mathrm{HA}-\mathrm{StrepII}$ (A) or $\mathrm{Nt} \mathrm{CDPK}_{2}-\mathrm{His}_{6}$ - $^{-}$ HA (B) on StrepTactin Sepharose or TALON resin, respectively, were separated by SDS-PAGE and analysed by Coomassie and silver stains or Western blot (anti-HA antibody). Marker proteins (M) are indicated with their molecular masses in $\mathrm{kDa} .10 \mu \mathrm{l}$ of each fraction were applied per lane. To allow quantitative comparisons, the total volume of each fraction (in $\mathrm{ml}$ ) are given at the bottom of the Coomassie-stained gels. Arrows indicate the purified $N t$ CDPK2 protein. Lanes 1, extract before the addition of resin (input); lanes 2, extract after the incubation with resin (unbound protein); lanes 3, resin after washes, before elution (bound protein); lanes 4, affinity eluted protein, pool 1 (purified protein); lanes 5, affinity eluted protein, pool 2; lanes 6, resin after elution (protein remaining on resin); lanes 7 , as 4 but silverstained; lanes 8, affinity eluted protein (pool 1) from a control purification of GFP expressed in $N$. benthamiana. 
(A)

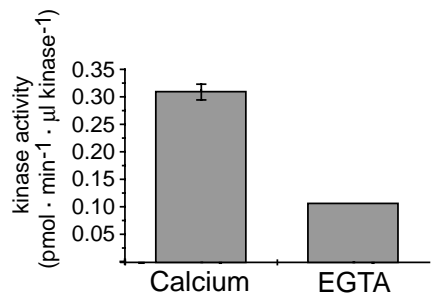

(B)

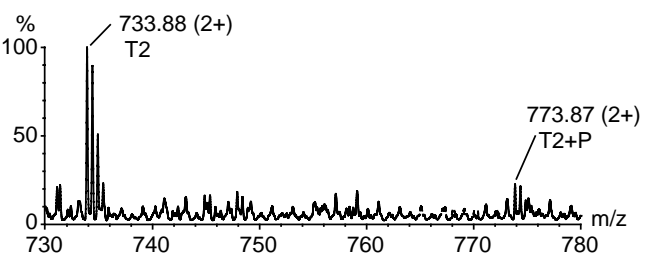

(C)

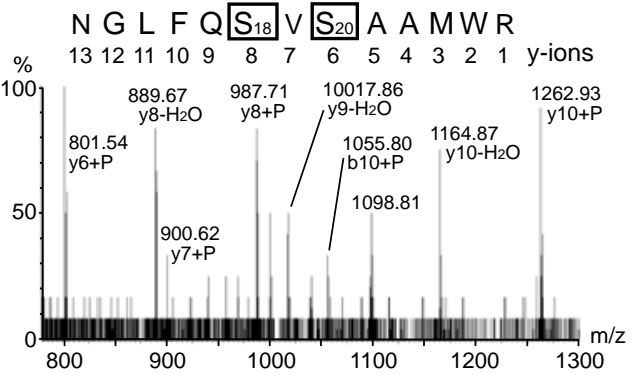

Figure 3. Enzymatic activity of $N t$ CDPK2-HA-StrepII and identification of in planta phosphorylation sites. NtCDPK2HA-StrepII was affinity purified from $N$. benthamiana using StrepTactin Sepharose. (A) Kinase activity was assayed by incorporation of ${ }^{32} \mathrm{P}$ into syntide- 2 in presence of $1 \mathrm{mM}$ calcium or $2 \mathrm{mM}$ EGTA and quantified by scintillation counting. (B) MS spectrum from 730 to $780 \mathrm{~m} / z$ displaying the doubly charged tryptic peptide T2 (amino acids 13-25) in either nonphosphorylated $(\mathrm{T} 2 ; 733.88 \mathrm{~m} / \mathrm{z})$ or phosphorylated $(\mathrm{T} 2+\mathrm{P}$; $773.87 \mathrm{~m} / \mathrm{z}$ ) form. (C) MS/MS spectrum from 780 to $1300 \mathrm{~m} / \mathrm{z}$ displaying fragment ions of the phosphorylated peptide T2P $(773.87 \mathrm{~m} / \mathrm{z})$. Masses are labeled in reference to the expected fragment masses for the non-phosphorylated peptide T2 with "-H2O" indicating a loss of 18 mass units and " $+\mathrm{P}$ " a gain of 80 mass units characteristic for phosphorylation. The sequence of T2 is given on top and the numbers of possible y-ions are indicated underneath. The potential phosphorylation sites S18 and S20 are marked by a box. Phosphorylation at S20 is indicated by the presence of $\mathrm{y}$-ions $6+\mathrm{P}$ and $7+\mathrm{P}$.

kinase activity when syntide-2, a peptide substrate generally used for CDPK characterisation, was used in the assay (Figure 3A). Proteolytic digest and subsequent nanospray quadrupole time-offlight mass-spectrometry (Q-TOF MS) analysis could be performed directly on the purified protein without the need for further electrophoresis and in-gel digestion. Due to the high degree of

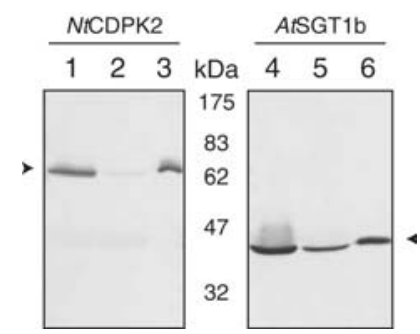

Figure 4. Improved binding yield of $N t \mathrm{CDPK} 2$ and $A t \mathrm{SGT} 1 \mathrm{~b}$ using the StrepTactin-Macroprep resin. NtCDPK2-HA-StrepII (left panel) and $A t$ SGT1b-StrepII (right panel) were expressed in $N$. benthamiana and purified using StrepTactin-Macroprep resin. Western blots were probed with StrepTactin-alkaline phosphatase conjugate. Molecular masses of marker proteins are indicated in $\mathrm{kDa}$. Lanes 1 and 4, extract before addition of the resin (input); lanes 2 and 5, extract after the incubation with resin (unbound protein); lanes 3 and 6 , protein on resin after washes (boiled off in SDS buffer). Of each fraction (total volume $0.8 \mathrm{ml}$, respectively) $10 \mu \mathrm{l}$ were applied per lane.

purity, mass spectra were dominated by peptides of $N t$ CDPK2 which facilitated the search for post-translational modifications. An example is given in Figure 3B showing a mass spectrum that contains peaks of a tryptic $N t$ CDPK2 peptide (amino acids 13-25) present in non-phosphorylated $(733.88 \mathrm{~m} / \mathrm{z})$ and phosphorylated $(773.87 \mathrm{~m} / \mathrm{z})$ form. Tandem mass-spectrometric analysis of the phosphorylated peptide clearly showed in planta phosphorylation of serine 20 demonstrated by the presence of phosphorylated $\mathrm{y}$-fragment ions $\mathrm{y} 6+\mathrm{P}$ and $\mathrm{y} 7+\mathrm{P}$ in the spectrum (Figure 3C). As a negative control for purification specificity, extracts from plants expressing smGFP were subjected to the same purification scheme. Nonspecific protein purification was not observed (Figure 2A, lane 8). Western analysis of extracts taken before and after incubation with the affinity matrix revealed that only part of the protein was bound to the StrepTactin resin (compare lanes 1 and 2). The bound fraction is estimated in lane 3 (protein boiled off the resin in SDS buffer after washes). Binding was not significantly increased by extending the incubation time or binding at higher temperatures (data not shown). Affinity elution using desthiobiotin was possible (lane 4) though not quantitative (lanes 5 and 6). Binding of StrepII-tagged proteins was greatly improved if instead of StrepTactin Sepharose an alternative matrix termed Strep Tactin Macroprep (IBA GmbH, Göttingen, Germany) was used (Figure 4, left panel, lanes 1 
and 2). Bound protein could be boiled off the column in SDS buffer (lane 3) but affinity elution from this alternative matrix was not possible in our hands. However, enzymatic activity of $N t$ CDPK2 was retained on the Macroprep resin and MS-analysis after gel-electrophoresis and ingel digest yielded spectra with a high density of peptide signals specific to the isolated protein (data not shown).

Despite widespread application of the His tag in Escherichia coli there are few reports on its use in plants (Terpe, 2003). One of the major drawbacks is its incompatibility with reductants and chelators. While chelators are generally used to block metal-proteases and may be dispensable, reductants like dithiothreitol (DTT) or $\beta$-mercaptoethanol are absolutely required while preparing a protein extract from most plant species to avoid rapid oxidation leading to enzyme inactivation (e.g. Witte and Medina-Escobar, 2001) and aggregation. To avoid these problems, extracts containing $\mathrm{N} t \mathrm{CDPK} 2-\mathrm{His}_{6}$-HA were first prepared in the presence of small amounts of DTT and chelators. Since the kinase is mostly associated with the microsomal pellet these compounds could then be washed away before extracting the pellet with detergent-containing buffer to solubilise the enzyme (see Materials and methods). For soluble proteins (such as AtSGT1b) a desalting step (dialysis or gel filtration) would be necessary after extraction with reductants and chelators before the extract could be chromatographed on an IMAC column. Although the microsomal pellet fraction applied to the IMAC column (TALON resin) was less complex than the total extract applied to the StrepTactin matrix (compare Figure 2A and B, lane 1) purity attained after IMAC purification was poor compared to the enrichment after StrepTactin chromatography (compare Figure 2A and B, lanes 4 or 7). Despite initially extracting in the presence of DTT partial oxidation could not be avoided, leading to the appearance of $\mathrm{N} t \mathrm{CDPK} 2$ aggregates visible as high molecular species of NtCDPK2 on the Western blot (Figure 2B). Binding yield to the IMAC column is comparable to that obtained with the StrepTactin Sepharose column (compare lanes 1-3 of Figure 2A and B).

Purification of $N t$ CDPK2 with the TAP-tag system was not attempted because we reasoned that the calmodulin-like domain of the CDPK would probably bind to the calmodulin-binding domain of the TAP tag and therefore interfere with the purification. This notion is supported by the finding that calmodulin can functionally replace the calmodulin-like domain in a hybrid CDPK (Yoo and Harmon, 1996).

\section{Use of avidin prevents co-purification of biotinylated proteins on StrepTactin}

StrepTactin is a streptavidin derivative which, like streptavidin, also binds free biotin and biotinylated proteins. Therefore, biotinylated proteins are co-purified with StrepII-tagged proteins (Figure 5, third panel, lanes 1 and 2: signals marked with asterisk) and binding sites on the StrepTactin matrix may be blocked by free biotin. This may be of particular relevance when working with plant extracts because free cytosolic biotin levels have been estimated at $11 \mu \mathrm{M}$ (in pea leaves), three orders of magnitude higher than in bacteria (Tissot et al., 1997). However, free and protein-bound biotin can be captured by inclusion of avidin in the extraction buffer, taking advantage of the fact that the StrepII tag does not bind to avidin (Skerra and Schmidt, 2000). We observed that this procedure blocked co-purification of biotinylated proteins (Figure 5, third panel) but did not diminish the binding of $N t$ CDPK2-HA-StrepII to StrepTactin (Figure 5, all panels). Additionally, one-step

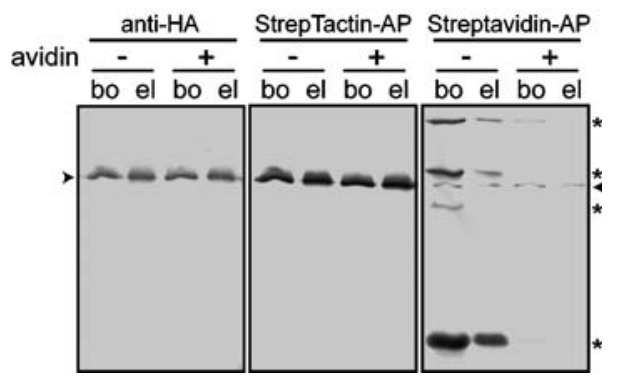

Figure 5. Effect of avidin on $N t$ CDPK2 purification. Western blots of $N t$ CDPK2-HA-StrepII purified on StrepTactin Sepharose in the presence $(+)$ or absence (-) of avidin. Detection was performed either with anti-HA primary antibody and anti-rat IgG-alkaline phosphatase conjugate (left panel), StrepTactin-alkaline phosphatase conjugate (middle panel) or with Streptavidin-alkaline phosphatase conjugate (right panel). bo: protein bound to the resin after washes, directly before elution (total fraction size $0.8 \mathrm{ml}$ ); el: affinity eluted protein (total fraction size $0.2 \mathrm{ml}$ ). An amount of $10 \mu \mathrm{l}$ of each fraction were applied per lane. Arrows indicate the purified $N t$ CDPK2StrepII and asterisk designate biotinylated proteins. 
detection of the StrepII tag with StrepTactinalkaline phosphatase conjugate (Figure 5, middle panel) produced an equivalent signal intensity on a Western blot compared to the conventional twostep HA-tag detection with primary and secondary antibodies (left panel).

Purification of AtSGT1b from $\mathrm{N}$. benthamiana using a StrepII tag or a TAP tag

$A t \mathrm{SGT1b}$ is a soluble protein with features of molecular co-chaperones that has been implicated in disease resistance and auxin responses of plants (Hubert et al., 2003; Muskett and Parker, 2003). AtSGT1b-StrepII complemented auxin insensitivity of the Ler sgt1b-3 mutant (Gray et al., 2003) in
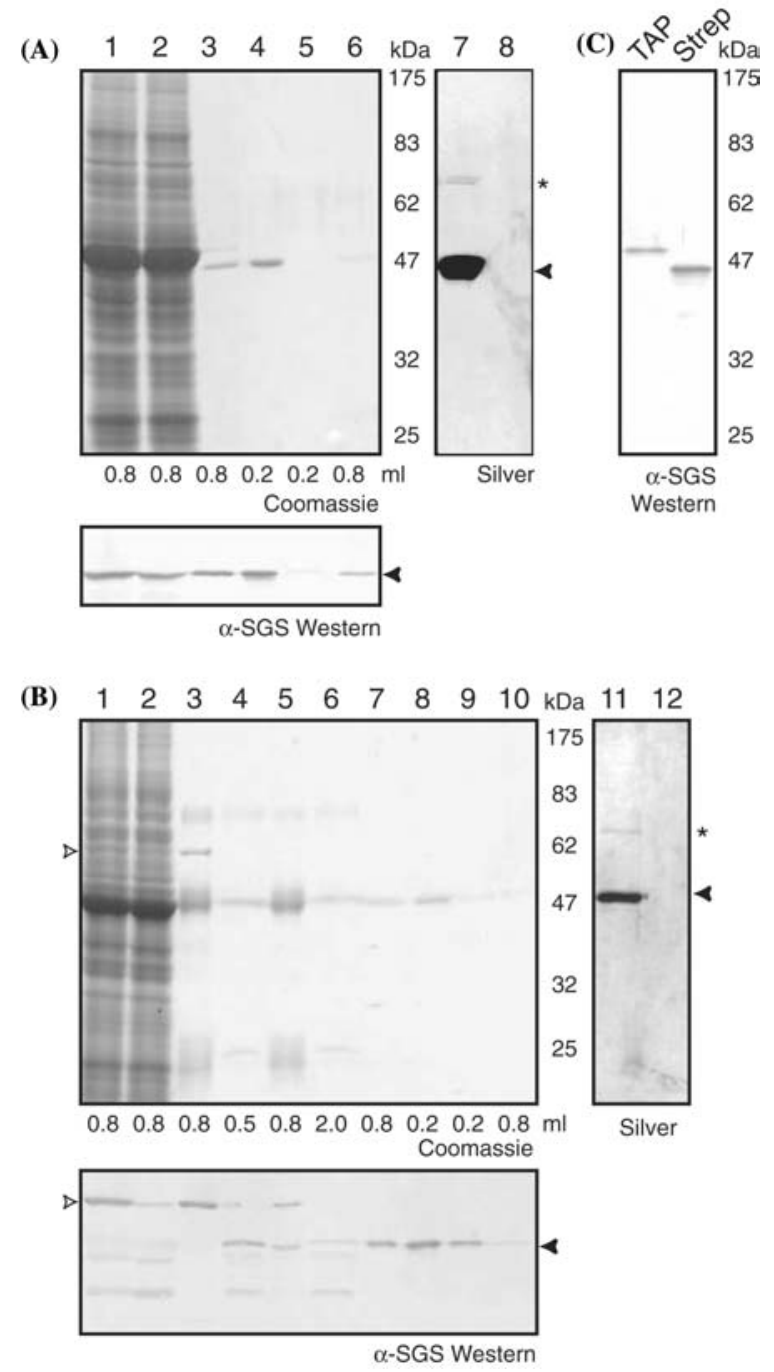

a root growth inhibition assay and therefore appears to possess biological activity (data not shown). An in vitro test for activity is currently not available.

As for $N t$ CDPK2, $A t$ SGT1b-StrepII expressed in $N$. benthamiana was purified to near homogeneity (Figure 6A, lanes 4 and 7). However, a protein of $\sim 70 \mathrm{kDa}$ was co-purified that was absent in the GFP control (lane 8) and therefore represents a possible $A t S G T 1 b$ interactor. Affinity elution from the StepTactin-Sepharose matrix was marginally more efficient for $A t \mathrm{SGT} 1 \mathrm{~b}$ than for the $N t$ CDPK2 (compare lanes 4-6 of Figures 2A and 6A). Similar to our findings with $N t$ CDPK2, binding yield of AtSGT1b could be improved using a StrepTactin-Macroprep resin (Figure 4, right panel).

The TAP tag was first used to purify protein complexes from yeast (Rigaut et al., 1999). A

Figure 6. Purification of AtSGT1b from N. benthamiana via StrepII tag and TAP tag. The different fractions collected during purification of AtSGT1b-StrepII using StrepTactin Sepharose (A) or AtSGT1b-TAP (B) were separated by SDS-PAGE and analysed by Coomassie and silver stains or Western blot (anti-SGS antibody). Molecular masses of marker proteins are indicated in $\mathrm{kDa}$. An amount of $10 \mu \mathrm{l}$ of each fraction were applied per lane. To allow quantitative comparisons, the total volume of each fraction (in $\mathrm{ml}$ ) is given at the bottom of the Coomassie-stained gels. White and black arrows indicate full-length $A t \mathrm{SGT} 1 \mathrm{~b}-\mathrm{TAP}$ and purified $A t \mathrm{SGT} 1 \mathrm{~b}$, respectively. Note that TEV protease cleavage leads to a reduction in protein size. The asterisk designates a protein that co-purified with AtSGT1b. (A) Lane 1, extract before the addition of resin (input); lane 2, extract after the incubation with resin (unbound protein); lane 3 , resin after washes, before elution (bound protein); lane 4, affinity eluted protein, pool 1 (purified protein); lane 5, affinity eluted protein, pool 2; lane 6, resin after elution (protein remaining on resin); lane 7, as lane 4 but silver-stained; lane 8, affinity eluted protein (pool 1) from a control purification of GFP expressed in $N$. benthamiana. (B) Lane 1, extract before the addition of IgG resin (input); lane 2, extract after the incubation with IgG resin (unbound protein); lane $3, \mathrm{IgG}$ resin after washes, before elution (bound protein); lane 4, TEV-eluted protein; lane 5, IgG resin after elution (protein remaining on resin); lane 6, extract after the incubation with calmodulin resin (unbound protein); lane 7, calmodulin resin after washes, before elution (bound protein); lane 8, EGTA-eluted protein, pool 1 (purified protein); lane 9, eluted protein, pool 2; lane 10, calmodulin resin after elution (protein remaining on resin); lane 11, as lane 8 but silver stained; lane 12 , affinity eluted protein, pool 1 , from a control purification of GFP expressed in $N$. benthamiana. C. AtSGT1b-TAP (lane 1, marked TAP) and AtSGT1b-StrepII (lane 2, marked Strep) protein amounts in purified fractions were compared by Western analysis (anti-SGS antibody). 
recent report describes the use of a modified TAP tag for the purification of proteins from plants (Rohila et al., 2004). In our hands, the TAP tag allowed isolation of $A t \mathrm{SGT} 1 \mathrm{~b}$ to the same degree of purity as observed for the StrepII-tagged protein (Figure 6B, lanes 8 and 11), with similar overall yield (Figure 6C). However, starting from the crude extract StrepII-tag purification took less than 60 min while $5 \mathrm{~h}$ were needed for a TAP-tag purification. As for the AtSGT1b-StrepII purification, a protein of $\sim 70 \mathrm{kDa}$ was co-purified (lane 11 of Figure $6 \mathrm{~B}$ ). Binding to the $\mathrm{IgG}$ column was almost quantitative (compare lanes 1-3 of Figure $6 \mathrm{~B}$, Western). During cleavage with the TEV protease $\left(2 \mathrm{~h}\right.$ at $\left.16^{\circ} \mathrm{C}\right)$ some $A t \mathrm{SGT} 1 \mathrm{~b}$ was degraded (Figure 6B, lane 4, Western), presumably by the action of co-purified proteases from the plant extract. Not all $A t$ SGT1b-TAP was released from the $\operatorname{IgG}$ column (lane 5). Binding of the eluted $A t$ SGT1b to the calmodulin column was nearly complete (compare lanes 4 and 6, Western; and see binding yield in lane 7) and the protein could be eluted quantitatively (lanes 8-10).

Purification of $A t$ SGT1b using a His tag was not attempted due to the soluble nature of the protein. Removal of chelators and reducing agents prior to affinity chromatography would have required additional steps like desalting gel filtration or dialysis. This was not compatible with our aim to find an efficient purification method.

\section{Purification of AtSGT1b via StrepII tag or TAP tag} from transgenic $\mathrm{A}$. thaliana plants

We also investigated the use of the StrepII and TAP tags for $A t S G T 1 b$ purification from transgenic A. thaliana. Non-transformed Ler sgtlb-3 mutant served as control. Purification of $A t$ SGT1b-StrepII and $A t$ SGT1b-TAP from A. thaliana was achieved to high purity (Figure 7) similar to our results obtained with $N$. benthamiana. Q-TOF MS analysis of purified AtSGT1bStrepII covering $73 \%$ of the whole protein did not provide evidence for any post-translational modification (data not shown). With both tagged variants, a protein of $\sim 70 \mathrm{kDa}$ was co-purified at sub-stoichiometric levels and, using protein purified via the StrepII tag, was identified after in-gel digestion by nanospray Q-TOF MS analysis (L.D. Noël et al., unpublished). A higher amount of $A t$ SGT1b-TAP (and co-purifying $70 \mathrm{kDa}$

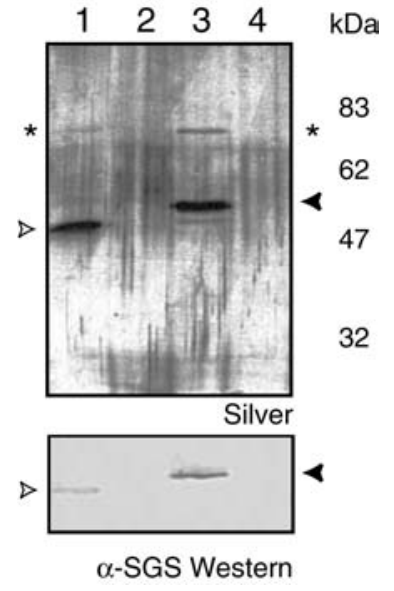

Figure 7. Purification of AtSGT1b and a potential interacting protein from $A$. thaliana extracts via StrepII tag and TAP tag. Extracts from Ler sgt 1b-3 expressing AtSGT1b-StrepII (lane 1) and AtSGT1b-TAP (lane 3) and non-transgenic Ler sgt1b-3 (control, lanes 2 and 4) were subjected to the respective purification schemes. Purified proteins were analysed by SDS-PAGE and detected by silver staining (upper panel) or Western blot (anti-SGS antibody, lower panel). An amount of $10 \mu \mathrm{l}$ of the final elution fractions (pool 1) were loaded. White and black arrows indicate the purified AtSGT1b-StrepII and AtSGT1b-TAP proteins, respectively. The asterisk indicates the position of a protein that co-purified with AtSGT1b. Molecular masses of marker proteins are indicated in $\mathrm{kDa}$.

protein) than AtSGT1b-StrepII was obtained (Figure 7) which may have been due to different amounts of tagged proteins in the corresponding crude extracts. An in-depth characterization of $A t$ SGT1b biological function and interactions is currently being undertaken and will be presented elsewhere. In summary, our data suggest that the TAP- as well as the StrepII-epitope are suitable for the purification of protein complexes from plants.

\section{Discussion}

The pace of plant gene discovery and transcription analyses has increased massively in recent years, affording new insights to the roles of genes and their products. As a first resort, plant biologists generally attempt production of recombinant plant proteins in E. coli or yeast cells for biochemical analysis. Such studies can provide important information about intrinsic biochemical properties. There is, however, an increasing need to unravel the nature of in vivo regulatory controls and modifications influencing protein function. Often these 
questions can only be addressed by isolation of proteins and protein complexes from their natural plant source. Ideally such isolation should be rapid and yielding protein of high purity.

In this study we report on the successful use of the StrepII and TAP tags for protein purification from plant extracts. The utility of a modified TAP tag for plant work has been shown recently (Rohila et al., 2004). Both tags allowed the isolation of $N t \mathrm{CDPK} 2$ and $A t \mathrm{SGT} 1 \mathrm{~b}$ to high purity (Figures 2A and 6). In contrast, several plant proteins appeared to co-purify during isolation of $\mathrm{Nt}$ CDPK2-His ${ }_{6}$ on an IMAC column (Figure 2B), presumably because they contained metal-binding or natural His-tag sequences like, for example, those observed in urease accessory protein G (Witte et al., 2001). Purity achieved by the StrepIItag system was further increased by the inclusion of avidin in the extraction buffer to suppress copurification of biotinylated proteins (Figure 5). Mass spectra of the StrepII-isolated proteins were dominated by peptides of either AtSgtlb or $N t$ CDPK2 which facilitated the search for posttranslational modifications. Serine 20 of $N t$ CDPK 2 was identified as an in planta phosphorylation site (Figure 3C). Tandem mass spectra were also consistent with the possibility that a minor part of peptide T2 may be alternatively phosphorylated at S18 as previously reported for recombinant kinase produced in E. coli (Glinski et al., 2003). There are, to our knowledge, very few examples where in vivo phosphorylation was directly demonstrated for a particular plant protein under study. For eukaryotic organisms it was estimated that about one-third of all proteins are subject to phosphorylation (Mann et al., 2002) and the regulatory role of such post-translational modifications is widely appreciated. Unravelling the locations and functions of these post-translational events in plant proteins may be facilitated by the protocols described here.

Purification via the TAP tag required approximately $5 \mathrm{~h}$ due to the combination of two purification steps and the need for an intermediate protease cleavage which alone takes $2 \mathrm{~h}$ (without reaching completion; Figure 6B). In contrast, the StrepII purification procedure could be performed in less than 60 min, likely reducing the risk of protein degradation, loss of interacting proteins or alteration of informative post-translational modifications. Additionally, the shorter processing time for StrepII-tagged proteins allowed us to proceed from the plant leaf to the MS analysis in one day.

The StrepII tag comprises only eight amino acids and has no particular overall charge, unlike the positively charged $\mathrm{His}_{6}$ tag. The TAP tag codes for 181 amino acids. In principle, the StrepII tag is therefore least likely to interfere with the biological function of the tagged protein. This may be a particular advantage if the aim is to isolate protein complexes in a non-mutant background since proteins with bulky tags may be less efficient in competing with the endogenous protein for binding partners. This problem has been realised for the TAP tag and an approach employing double stranded RNA interference to deplete endogenous proteins in combination with the expression of TAP-tagged forms was proposed (Forler et al., 2003).

In terms of flexibility the StrepII tag has few obvious limitations. In principle, any buffer or buffer additive that suits protein stability may be used (with the exception of biotin). In contrast, IMAC is incompatible with chelators and reducing agents (Chaga, 2001) that are indispensable for extracting proteins from many plant species (Gegenheimer, 1990). The TAP tag limits the use of protease inhibitors that block TEV cysteine protease activity. The absence of inhibitors may cause degradation problems during the two-hour incubation with TEV protease at $16{ }^{\circ} \mathrm{C}$ (see Figure 6B, lane 4) presumably due to the action of co-purified plant proteases. However, a recent report indicated that the inclusion of the cysteine protease inhibitor E-64 improved the stability of a TAP-tagged protein during TEV protease cleavage (Rohila et al., 2004). Whether this inhibitor is of general utility for different proteins and for purifcations from plant sources other than Arabidopsis remains to be shown. During TAP-tag purification, chelating agents are needed for the elution from the calmodulin column. Thus, purification of metal-containing enzymes is problematic because metal ions needed for biological function may be stripped from the purified protein.

Overall yield (amount of tagged protein present in the crude extract versus amount of tagged protein recovered in the purified fraction) remains a hurdle for all the tested tagging systems including the StrepII system. Using a different StrepIImatrix (Macroprep instead of Sepharose) may be a 
solution in some cases (Figure 4). Although we could not achieve affinity elution from the Macroprep resin, MS-analysis is still possible when the protein is boiled off from the resin in SDS buffer, electrophoresed and digested in-gel. Due to the high binding yield on the Macroprep resin this procedure even facilitates MS analysis because protein concentrations are higher. Furthermore, strong binding may be desirable for applications using immobilised protein. Overall yield of TAPtagged $A t$ SGT1b protein appeared to be slightly inferior to that of StrepII-tagged AtSGT1b when purified from $N$. benthamina (Figure 6C). The situation was reversed for purification of these tagged $A$ tSGT1b derivatives from $A$. thaliana (Figure 7). Such yield differences may reflect differences in the amount of tagged protein present in the respective crude extracts. This is likely for extracts obtained from $A$. thaliana since distinct transgenic lines expressing the respective tagged proteins were used. Due to the presence of protein A in the TAP tag it was not possible to compare accurately amounts of StrepII-tagged versus TAPtagged protein in the corresponding crude extracts by Western blot analysis. Taken together, our data indicate that yield from the TAP-tag and the StrepII-tag system is similar. Losses in the TAPtag system do not appear to result from low binding yield to the two resins, rather from degradation during TEV protease cleavage and incomplete release of protein from the IgG column (Figure 4B). The combination of two purification steps, each associated with some losses, also reduces overall recovery.

In the course of this study we also tested the HAT tag (BD biosciences Clontech, Palo Alto, CA), a 19 amino acid tag for use in IMAC chromatography (NtCDPK2-HA-HAT construct) and the results obtained were similar to those with the $\mathrm{His}_{6}$ tag (data not shown). In addition, preliminary experiments demonstrated that the 15-amino acid $\mathrm{S}$ tag (Raines et al., 2000) and the chitin-binding-domain (CBD) tag (52 aa; Xu et al., 2000) also allowed purification of $N t$ CDPK 2 from crude plant extracts. However, elution of S-tagged protein was only possible with high amounts of salt $\left(3 \mathrm{M} \mathrm{MgCl}_{2}\right)$ while affinity elution of CBDtagged protein was not possible (C.-P. Witte and T. Romeis, unpublished). Tags employing recognition by antibodies were not tested due to the high cost and limited stability of antibody matrices
(Terpe, 2003). As StrepTactin is derived from Streptavidin, a protein of high stability (Bayer et al., 1990), it is likely to be comparatively stable. According to the manufacturer, stripping and recycling the resin is possible. At the current price of the StrepTactin-Sepharose matrix, protein purification applying this system should be affordable to most laboratories and may be suitable for large-scale or high-throughput applications (Boettner et al., 2002; Holz et al., 2002).

\section{Acknowledgements}

We are grateful to Paul Schulze-Lefert, Laurent Deslandes and the Romeis and Parker group members for critical comments. This work was supported by the Max Planck Society, the Alexander von Humboldt Foundation, the Deutsche Forschungsgemeinschaft (SFB 635) and Alexander von Humboldt and EMBO long term fellowships to L.D.N and C.-P.W., respectively.

\section{References}

Austin, M.J., Muskett, P., Kahn, K., Feys, B.J., Jones, J.D. and Parker, J.E. 2002. Regulatory role of SGT1 in early R genemediated plant defenses. Science 295: 2077-2080.

Azevedo, C., Sadanandom, A., Kitagawa, K., Freialdenhoven, A., Shirasu, K. and Schulze-Lefert, P. 2002. The RAR1 interactor SGT1, an essential component of R gene-triggered disease resistance. Science 295: 2073-2076.

Bayer, E.A., Benhur, H. and Wilchek, M. 1990. Isolation and properties of streptavidin. Meth. Enzymol. 184: 80-89.

Boettner, M., Prinz, B., Holz, C., Stahl, U. and Lang, C. 2002. High-throughput screening for expression of heterologous proteins in the yeast Pichia pastoris. J. Biotechnol. 99: 51-62.

Chaga, G.S. 2001. Twenty-five years of immobilized metal ion affinity chromatography: Past, present and future. J. Biochem. Biophys. Methods 49: 313-334.

Cheng, S.H., Willmann, M.R., Chen, H.C. and Sheen, J. 2002. Calcium signaling through protein kinases. The Arabidopsis Calcium-Dependent Protein Kinase gene family. Plant Physiol. 129: 469-485.

Clough, S.J. and Bent, A.F. 1998. Floral dip: A simplified method for Agrobacterium-mediated transformation of A. thaliana. Plant J. 16: 735-743.

Davis, S.J. and Vierstra, R.D. 1996. Soluble derivatives of green fluorescent protein (GFP) for use in A. thaliana. Weeds World 3: 43-48.

Forler, D., Kocher, T., Rode, M., Gentzel, M., Izaurralde, E. and Wilm, M. 2003. An efficient protein complex purification method for functional proteomics in higher eukaryotes. Nat. Biotechnol. 21: 89-92.

Gegenheimer, P. 1990. Preparation of Extracts from Plants. Meth. Enzymol. 182: 174-193. 
Glinski, M., Romeis, T., Witte, C.-P., Wienkoop, S. and Weckwerth, W. 2003. Stable isotope labeling of phosphopeptides for multiparallel kinase target analysis and identification of phosphorylation sites. Rapid Commun. Mass Spectrom. 17: 1579-1584.

Gray, W.M., Muskett, P.R., Chuang, H.W. and Parker, J.E. 2003. Arabidopsis SGT1b is required for SCF(TIR1)mediated auxin response. Plant Cell 15: 1310-1319.

Holz, C., Hesse, O., Bolotina, N., Stahl, U. and Lang, C. 2002. A micro-scale process for high-through put expression of cDNAs in the yeast Saccharomyces cerevisiae. Protein Expres. Purif. 25: 372-378.

Hubert, D.A., Tornero, P., Belkhadir, Y., Krishna, P., Takahashi, A., Shirasu, K. and Dangl, J.L. 2003. Cytosolic HSP90 associates with and modulates the Arabidopsis RPM1 disease resistance protein. EMBO J. 22: 5679-5689.

Koncz, C. and Schell, J. 1986. The promoter of the TL-DNA gene 5 controls the tissue-specific expression of chimeric genes carried by a novel type of Agrobacterium binary vector. Mol. Gen. Genet. 204: 383-386.

Kornberg, A. 2003. Ten commandments of enzymology, amended. Trends Biochem. Sci. 28: 515-517.

Mann, M., Ong, S.E., Grønborg, M., Steen, H., Jensen, O.N. and Pandey, A. 2002. Analysis of protein phosphorylation using mass spectrometry: Deciphering the phosphoproteome. Trends Biotechnol. 20: 261-268.

Muskett, P. and Parker, J. 2003. Role of SGT1 in the regulation of plant R gene signalling. Microbes Infect. 5: 969-976.

Puig, O., Caspary, F., Rigaut, G., Rutz, B., Bouveret, E., Bragado-Nilsson, E., Wilm, M. and Seraphin, B. 2001. The tandem affinity purification (TAP) method: A general procedure of protein complex purification. Methods 24: 218-229.

Raines, R.T., McCormick, M., Van Oosbree, T.R. and Mierendorf, R.C. 2000. The S center dot tag fusion system for protein purification. Meth. Enzymol. 326: 362-376.

Rigaut, G., Shevchenko, A., Rutz, B., Wilm, M., Mann, M. and Seraphin, B. 1999. A generic protein purification method for protein complex characterization and proteome exploration. Nat. Biotechnol. 17: 1030-1032.

Rohila, J.S., Chen, M., Cerny, R. and Fromm, M.E. 2004. Improved tandem affinity purification tag and methods for isolation of protein heterocomplexes from plants. Plant J. 38: 172-181.

Romeis, T., Ludwig, A.A., Martin, R. and Jones, J.D.G. 2001. Calcium-dependent protein kinases play an essential role in a plant defence response. EMBO J. 20: 5556-5567.

Shevchenko, A., Wilm, M., Vorm, O. and Mann, M. 1996. Mass spectrometric sequencing of proteins from silver stained polyacrylamide gels. Anal. Chem. 68: 850-858.

Shevchenko, A., Chernushevich, I., Wilm, M. and Mann, M. 2000. De Novo Peptide Sequencing by Nanoelectrospray Tandem Mass Spectrometry Using Triple Quadrupole and Quadrupole/Time-of-Flight Instruments. In: J.R. Chapman (Ed.), Methods in Molecular Biology, Vol. 146, Humana Press, pp. 1-16.

Skerra, A. and Schmidt, T.G.M. 2000. Use of the Strep-tag and streptavidin for detection and purification of recombinant proteins. Meth. Enzymol. 326: 271-304.

Terpe, K. 2003. Overview of tag protein fusions: From molecular and biochemical fundamentals to commercial systems. Appl. Microbiol. Biot. 60: 523-533.

Tissot, G., Douce, R. and Alban, C. 1997. Evidence for multiple forms of biotin holocarboxylase synthetase in pea (Pisum sativum) and in A. thaliana: Subcellular fractionation studies and isolation of a cDNA clone. Biochem. J. 323: 179-188.

Voinnet, O., Rivas, S., Mestre, P. and Baulcombe, D. 2003. An enhanced transient expression system in plants based on suppression of gene silencing by the p19 protein of tomato bushy stunt virus. Plant J. 33: 949-956.

Witte, C.P. and Medina-Escobar, N. 2001. In-gel detection of urease with nitroblue tetrazolium and quantification of the enzyme from different crop plants using the indophenol reaction. Anal. Biochem. 290: 102-107.

Witte, C.P., Isidore, E., Tiller, S.A., Davies, H.V. and Taylor, M.A. 2001. Functional characterisation of urease accessory protein G (ureG) from potato. Plant Mol. Biol. 45: 169-179.

Xu, M.Q., Paulus, H. and Chong, S.R. 2000. Fusions to selfsplicing inteins for protein purification. Meth. Enzymol. 326: 376-418.

Yoo, B.-C. and Harmon, A.C. 1996. Intramolecular binding contributes to the activation of CDPK, a protein kinase with a calmodulin-like domain. Biochemistry 35: 12029-12037. 